\section{Lohnt sich bei Zervixkarzinom das operative Staging vor der Radiochemotherapie?}

\begin{abstract}
Ziel der Studie Uterus 11 war es, die Bedeutung eines operativen, besonders eines laparoskopischen, Stagings vor der Radiochemotherapie bei Patientinnen mit einem Zervixkarzinom im FIGO-Stadium IIB-IVA zu untersuchen.
\end{abstract}

\begin{abstract}
D ie primäre Radiochemotherapie ist bei Patientinnen mit lokal fortgeschrittenem Zervixkarzinom als Erstlinientherapie etabliert, kann aber mit frühen und späten Toxizitäten einhergehen. Gemäß FIGO-Klassifikation ist das klinische Staging Standard. Über den Nutzen des operativen Stagings wird nach wie vor diskutiert. Es wurde berichtet, dass beim operativen Lymphknoten-Staging oftmals eine Hochstufung erforderlich ist. In der prospektiven, randomisierten, multizentrischen Studie wurden 255 Patientinnen mit einem Zervixkarzinom (FIGO IIB-IVA) randomisiert dem operativen (Arm A) oder dem klinischen Staging (Arm B), jeweils mit nachfolgender Radiochemo-
\end{abstract}

therapie, zugeteilt. Für die Bestrahlung wurden intensitätsmodulierte (IMRT) und andere moderne Techniken empfohlen. Patientinnen mit paraaortalen Metastasen unterzogen sich einer erweiterten Bestrahlung („extended-field“). Die Brachytherapie war für alle obligatorisch. Als Chemotherapie wurde Cisplatin oder Carboplatin eingesetzt. 236 Patientinnen unterzogen sich einer Bestrahlung mit median 50,4 Gy, $60 \%$ wurden mit IMRT behandelt. Die mittlere Therapiedauer betrug 53 Tage. Das operative Staging führte bei mehr Patientinnen zu einer erweiterten Bestrahlung ( 23 vs. $12 \%$; p < 0,014). In Arm B erhielten signifikant mehr Patientinnen die volle geplante Chemotherapiedosis.
Die Therapie wurde gut vertragen. Es traten keine urogenitalen oder gastrointestinalen Toxizitäten vom Grad $\geq 3$ auf. Grad-3-Nebenwirkungen waren: Übelkeit (6\%), Erbrechen (3\%) und Diarrhö $(<2 \%)$. Im Arm A war der Anteil an Patientinnen mit einer Grad-2-Anämie $(54,3$ vs. $45,3 \% ; \mathrm{p}=0,074)$ und einer Grad-2-Leukozytopenie (41,4 vs. 31,6\%; $\mathrm{p}=0,56)$ höher als im Arm B.

Fazit: Das operative Staging führte zu einer Verdoppelung der Anzahl an Patientinnen, für die eine erweiterte Bestrahlung notwendig wurde. Die Frage, ob das operative Staging in Zusammenhang mit der primären Radiochemotherapie vorteilhaft ist, konnte jedoch aufgrund dieser Daten noch nicht beantwortet werden.

Judith Neumaier

Marnitz S et al. Role of surgical versus clinical staging in chemo-radiated FIGO stage IIB-IVA cervical cancer patients. - Acute toxicity and treatment quality of the Uterus-11 multicenter phase III Intergroup trial of the German Radiation Oncology Group (ARO) and the Gynecologic Cancer Group (AGO). Int J Radiat Oncol Biol Phys. 2016;94(2):243-53.

\section{Zervixkarzinom: Radiochemotherapie nach der Hysterektomie nur bei Lymphknotenbeteiligung}

\begin{abstract}
Für Patientinnen mit einem Hochrisiko-Zervixkarzinom wird derzeit nach der Hysterektomie eine adjuvante Radiochemotherapie empfohlen. Dem Nutzen dieser Strategie im Vergleich zur alleinigen Bestrahlung wurde nun retrospektiv nachgegangen.
\end{abstract}

\footnotetext{
D ie Nationale Krebsdatenbank der USA (National Cancer Database, NCDB) wurde nach Frauen mit einem Zervixkarzinom durchsucht, die sich zwischen 2002 und 2012 einer Hysterektomie und einer adjuvanten Bestrahlung unterzogen hatten. Es wurden nur Patientinnen aufgenommen, die mindestens eines der folgenden Hochrisikokriterien (Peters-Kriterien) aufwiesen: Lymphknotenbeteiligung, positive Operationsränder, parametriale Invasion.

Insgesamt 2.479 Patientinnen hatten nach der Hysterektomie eine adjuvante Radiochemotherapie erhalten, 574 eine alleinige Bestrahlung. Frauen, die jünger als 70 Jahre waren, bei denen die Diagno-
}

se nach 2007 gestellt worden war und die keine Adenokarzinom-Histologie oder eine Lymphknotenbeteiligung aufwiesen, erhielten häufiger einer Radiochemotherapie als andere Patientinnen.

Die Radiochemotherapie verbesserte zwar das Gesamtüberleben in der gesamten Kohorte signifikant (Hazard Ratio [HR] 0,76; $p=0,022$ ). Detailliertere Analysen zeigten aber, dass die Radiochemotherapie das Gesamtüberleben lediglich bei Patientinnen signifikant verbesserte, die als einziges Peters-Kriterium eine Lymphknotenbeteiligung aufwiesen (HR $0,58, p=0,002)$. Patientinnen, die keine Lymphknotenbeteiligung, aber positive Operationsränder $(\mathrm{p}=0,73)$ oder eine pa- rametriale Invasion $(p=0,95)$ oder eine Kombination dieser beiden Faktoren aufwiesen ( $p=0,63)$, profitierten dagegen im Hinblick auf das Gesamtüberleben nicht von der Radiochemotherapie.

Fazit: Die adjuvante Radiochemotherapie nach der Hysterektomie verbessert das Gesamtüberleben bei Patientinnen mit einem Hochrisiko-Zervixkarzinom im Vergleich zur alleinigen Bestrahlung, aber dieser Vorteil scheint auf Patientinnen mit Lymphknotenbeteiligung beschränkt zu sein. Der Nutzen der adjuvanten Radiochemotherapie im Vergleich zur alleinigen Bestrahlung bei Patientinnen mit parametrialer Invasion und/oder positiven Operationsrändern (ohne Lymphknotenbeteiligung) sollte in prospektiven Studien weiter untersucht werden.

Judith Neumaier

Trifiletti DM et al. Postoperative Chemoradiation Therapy in High-Risk Cervical Cancer: Re-evaluating the Findings of Gynecologic Oncology Group Study 109 in a Large, Population-Based Cohort. Int J Radiat Oncol Biol Phys. 2015;93(5):1032-44. 